\section{P019 AUDIT ON THE MANAGEMENT OF SEXUAL HEALTH NEEDS OF YOUNG PEOPLE IN AN INTEGRATED COMMUNITY BASED SERVICE}

Sameer Ghodsara, Nisha Pal*, Matthew Hamill. Berkshire Healthcare Foundation Trust, slough, UK

\subsection{6/sextrans-2016-052718.74}

Introduction Our community based integrated service caters to a diverse population within the Slough area with pockets of high deprivation and rising rates of CSE. It is of paramount importance that clinicians are able to identify and risk assess those vulnerable have a valuable tool for documentation.

Aims and Objectives This audit was based on the BASHH standards on "Management of STIs and related conditions in children and young people (BASHH 2010)"

Methods Retrospective data collection of the first 100 new patients who accessed the service from July - Aug 2014 aged 18 and under from all three sites. The standards of the audit are: Offer of full STI screen (CT, GC, HIV, STS) to sexually active young people-100\%. Offered an STI screen-90\%. Completions of CSE risk assessment proforma- 100\%. Documentation of decision for referral (100\% of under 13 s, $90 \%$ of those aged 16 and under)-100-90\%.

Results $75 \%$ of the attendees were girls and had primarily contraception needs.STI screening was offered to all however the uptake of a full screen was less than 50\%. Overall documentation was less than satisfactory and decision to refer was documented in only $15 \%$.

Conclusions There are high rates of STI's among young people and risk taking behaviour was noticed in the attendances. Poor attendances among boys and MSM were identified. 50\% refused to have screening for BBV and the need of alternative testing methods like the saliva testing was highlighted. A CSE proforma was introduced and all referrals are discussed with the safeguarding lead and audited on a regular basis.

\section{P020 EVALUATING PATIENT PERSPECTIVES ON CONFIDENTIALITY IN SEXUAL HEALTH CLINICS}

${ }^{1}$ Sian Warren*, ${ }^{1}$ Georgina Forbes, ${ }^{2}$ Emily Souter. ${ }^{1}$ Dept Sexual Health, Cardiff Royal Infirmary, Cardiff, UK; ${ }^{2}$ Cardiff Medical School, Cardiff University, Cardiff, UK

\subsection{6/sextrans-2016-052718.75}

Background/introduction Since the introduction of the Venereal Diseases Act in 1917, confidentiality has been a key part of sexual health clinics in the UK. With the repealing of the VD Act, we devised a service evaluation to determine the patient's perspectives on confidentiality.

Aim(s)/objectives To determine patient understanding of the confidentiality process in a large inner city integrated sexual health (ISH) clinic.

Methods A patient questionnaire was designed and given to ISH clinic patients between $18^{\text {th }}$ of May and $5^{\text {th }}$ of June 2015 .

Results 163 responses were obtained from the ISH clinic (49\% female, $51 \%$ male). $89 \%$ patients reported confidentiality to be important or very important when attending the ISH clinic, with $97 \%$ patients reporting confidentiality to be important or very important in the diagnosis of STI's. With regards to ISH offering a non-judgmental service; 95\% patients reporting this to be important or very important. $68 \%$ patients reported the importance of ISH clinic records being kept separate from GP and hospital records. $45 \%$ patients reported they would not attend their GP for STI testing due to a variety of reasons such as embarrassment, convenience and wanting to attend a specialised service.

Discussion/conclusion This study confirms that confidentiality in the diagnosis of STI's and the non-judgemental care that patients receive continues to be important to service users. These factors influence which services patients wish to access for sexual health needs. It is therefore essential that ISH services continue to provide this level of care.

\section{P021 ABSTRACT WITHDRAWN}

\section{P022 IS INTRAVAGINAL BORIC ACID AN ALTERNATIVE THERAPEUTIC OPTION FOR VAGINAL TRICHOMONIASIS?}

Nicola Thorley*, Jonathan Ross. University Hospitals Birmingham NHS Foundation Trust, Birmingham, UK

\subsection{6/sextrans-2016-052718.76}

Background/introduction Current national guidance recommends treating Trichomonas vaginalis (TV) infection with nitro-imidazole therapy. The high prevalence of TV, high rate of metronidazole resistance and limited tolerability to nitroimidazoles when treating TV, suggest that alternative treatment regimens are required. Intravaginal Boric acid (BA) pessaries are available and have been used to safely treat vulvo-vaginal candidiasis and bacterial vaginosis.

Aim(s)/objectives We aimed to review the evidence for the safety and efficacy of BA for the treatment of TV.

Methods We performed a systematic review, in accordance with Centre for Reviews and Dissemination methods, of the evidence for the use of BA as a topical treatment for TV.

Results No randomised controlled trials or case series were found. Case reports provided in vivo evidence that BA safely and effectively treated TV. These cases, in the setting of resistant TV or severe metronidazole allergy, were managed with combination treatment administered over a period of 4 weeks to 5 months using doses of boric acid ranging from $600 \mathrm{mg}$ once a day to $600 \mathrm{mg}$ twice a day. No studies assessed the efficacy of $\mathrm{BA}$ in uncomplicated TV infection. In vitro, low concentrations $(0.2 \%)$ of $\mathrm{BA}$ reduced the growth rate of $\mathrm{TV}$, whereas higher concentrations $(\geq 0.4 \%)$ were lethal to both laboratory TV strains and clinical isolates, providing evidence that the inhibitory effect of BA on TV is dose-dependent.

Discussion/conclusion BA is well-tolerated and has in vitro and in vivo activity against TV. There is limited evidence on the appropriate dosing schedule. There is need for further evaluation in a clinical trial.

\section{P023 'IT'S ALL ABOUT THE MONEY MONEY MONEY'? OPTIMISING BLOOD INVESTIGATION REQUESTS FOR HIV PATIENTS}

Daisy Ogbonmwan*, Nathan Sankar, Mayur Chauhan. New Croft Centre, Newcastle, UK

10.1136/sextrans-2016-052718.77 
Background/introduction In 2010 Quality Innovation Productivity and Prevention (QIPP) was introduced to enable the NHS to provide cost efficient services. The 2013 BHIVA Standards of Care include the need to provide quality cost effective care.

Aim(s)/objectives Our aims were to assess whether unnecessary blood tests were undertaken during routine HIV assessments; to ensure minimal patient disruption and cost stewardship.

Methods Standards were established using current BHIVA and HIV CRG CD4 blood monitoring guidelines. A retrospective audit was carried out on patients attending for a routine review between the $1^{\text {st }}$ of December 2014 and the $31^{\text {st }}$ of January 2015 who had been on treatment for at least three months. Laboratory medicine cost data was ascertained.

Results 41 patient's notes and HARS entries were reviewed, $71 \%, 90 \%$, and $83 \%$ had their CD4 count, full blood count and lipids, respectively, unnecessarily requested. 44\%, 39\%, 56\% of the Syphilis, Hepatitis B and Hepatitis C blood tests respectively, were either not done as per the standards or inappropriately requested. There was a potential cost saving of over $£ 1300$ on blood tests where over 30\% were unnecessarily requested.

Discussion/conclusion Blood monitoring should not be a tick box exercise. Requesting unnecessary blood tests is not only costly but minor changes in the results may lead to unnecessary patient intervention. Clinic proformas can be used as an aid to whether investigations are required. Testing for Syphilis, Hepatitis B and C outside of the recommended standards should be guided by sexual histories taken during consultation.

\section{P024 CLINICAL CHARACTERISTICS OF HERPES SIMPLEX VIRUS URETHRITIS COMPAERD WITH CHLAMYDIA URETHRITIS AMONG MEN: A CASE CONTROL STUDY}

\begin{abstract}
1,3Jason Ong, 'Anna Morton*, ${ }^{1}$ Helen Henzel, ${ }^{1}$ Karen Berzins, ${ }^{2} J u l i a n$ Druce, ${ }^{1,3}$ Christopher Fairley, ${ }^{1,3}$ Catriona Bradshaw, ${ }^{4}$ Jane Hocking, ${ }^{1,3}$ Marcus Chen. ${ }^{1}$ Melbourne Sexual Health Centre, Melbourne, Australia; ${ }^{2}$ Victorian Infectious Disease Reference Laboratory, Melbourne, Australia; ${ }^{3}$ Central Clinical School, Monash University, Melbourne, Australia; ${ }^{4}$ Melbourne School of Population and Global Health, Melbourne, Australia
\end{abstract}

\subsection{6/sextrans-2016-052718.78}

Background/introduction Non-gonococcal urethritis (NGU) in males is a sexually transmitted infection commonly caused by Chlamydia trachomatis and Mycoplasma genitalium. Herpes simplex virus (HSV) has been reported as a causative agent in NGU; however, little is known about its clinical characteristics.

Aim(s)/objectives The study compared the clinical characteristics of men with HSV urethritis to those in men with chlamydial urethritis, and determined if there were any key differences.

Methods A retrospective case control study comparing the clinical and laboratory findings from men diagnosed with PCR confirmed HSV urethritis with those diagnosed with PCR confirmed chlamydial urethritis, was conducted between 2000 to 2015.

Results Eighty HSV urethritis cases were identified: 68\% (95\% CI 58-78) were HSV type 1 and 32\% (95\% CI 22-42) were HSV type 2. Compared with chlamydial urethritis, men with HSV urethritis were significantly more likely to report severe dysuria $(20 \%$ vs $0 \%, \mathrm{p}<0.01)$ or constitutional symptoms $(15 \%$ vs $0 \%, \mathrm{p}<0.01)$ and significantly less likely to report urethral discharge ( $19 \%$ vs $54 \%, \mathrm{p}<0.01)$. Men with HSV urethritis were significantly more likely to have meatitis $(62 \%$ vs $23 \%$, $\mathrm{p}<0.01)$, genital ulceration $(37 \%$ vs $0 \%, \mathrm{p}<0.01)$, and inguinal lymphadenopathy $(30 \%$ vs $0 \%, \mathrm{p}<0.01)$.
Discussion/conclusion In our study men with HSV urethritis had distinctive clinical features, not usually associated with chlamydial urethritis: severe dysuria, constitutional symptoms, meatitis, genital ulceration and lymphadenopathy. Clinicians should consider HSV when these are present.

\section{P025 IMPROVING LOCAL SEXUAL HEALTH SERVICES FOR LESBIAN, GAY, BISEXUAL AND TRANS (LGBT) PEOPLE}

Sinead Cook, Rachel Drayton*. Cardiff and Vale University Health Board, Cardiff, UK

\subsection{6/sextrans-2016-052718.79}

Background/introduction LGBT people have different care needs to other sexual health clinic attendees. Many STIs are known to disproportionately affect men who have sex with men (MSM). We wished to ascertain how to optimise LGBT, and in particular MSM, service provision by our urban sexual health clinic.

Methods 100 questionnaires were completed by attendees to the local Pride event.

Results 61 respondents self-defined as female, 34 male, 4 transman and 1 demifem. 27 (20 women, 4 men) stated their sexual partners were both male and female, 38 (12 women, 25 men) had same-sex partners, and 34 (28 women, 5 men) had opposite-sex partners only.

81 had not attended the local clinic. Reasons for this included previously living elsewhere (22), not feeling they required the service (15) or not knowing it existed (9). 67 reported they would like a specific LGBT sexual health clinic, with 63 requesting evening clinics. 9 did not want specific clinics, with 2 respondents citing concerns about discrimination. 61 felt more LGBT sexual health services outside the city centre are needed. Features they would like included web-based bookings (64), home-testing kits (49), pre-exposure prophylaxis (79) and HPV vaccination (69).

Discussion/conclusion The questionnaire was successful in capturing opinions of those who hadn't previously attended our service. However it is not possible to ascertain whether views expressed were representative of the local LGBT population as a whole and less than a third were MSM. We will consider developing a specific LGBT service in response to the survey's findings.

\section{P026 VALUE OF CONTINUING PHARYNGEAL GENPROBE APTIMA COMBO2 TRANSCRIPTION MEDIATED AMPLIFICATON (TMA) TESTING FOR CT/GC IN ADDITION TO UROGENITAL/RECTAL SWABS}

${ }^{1}$ Mrinalini Mahto*, ${ }^{2}$ Harry Mallinson. 'Macclesfield District Gen. Hospital, Macclesfield, Cheshire, UK; ${ }^{2}$ Aintree University Hospital, retired consultant Microbiology, Liverpool, UK

\subsection{6/sextrans-2016-052718.80}

Introduction BASHH guidelines say 'consider' Throat swabs (TS)/rectal swabs (RS) in females where history suggests \& to test in MSM. We were routinely testing females practicing fellatio \& MSM on throat swabs (TS) for CT/GC in addition to the genital/rectal sites.

Aim To review testing practices to look at whether TS gave extra positivity $\&$ whether it was cost effective. 